\title{
The Time Machine: A Multiscreen Generative Video Artwork
}

\author{
Daniel Buzzo \\ University of the West of England \\ Bristol, UK \\ Daniel.buzzo@uwe.ac.uk
}

\begin{abstract}
'The Time Machine' is a multi-screen, high-performance, generative video art installation based around multiple low cost computer platforms. Using algorithmic selection of palindromic loops of time-lapse video the work contrasts the external, machine perception of time with our internal, phenomenological experience of it. The video feeds, recorded from around the world, tick and tock backward and forward creating a polyrhythmic, 12 screen time-piece. The images loop back and forth on each screen of the installation, creating a large polyrhythmic clock of high definition, full colour motion. Each screen detailing a passage of time from around the world, captured, frozen, forward and reverse. The time-lapse loops slowly switch, selected from over a thousand separate pieces by generative algorithms on each host computer. Creating a Time Machine reflecting the world, gently rocking back and forth with a myriad of sub-cadences, confronting the viewer with the unanswerable challenge of comprehending time.
\end{abstract}

Time. Temporality. Media art. Generative video. Time lapse. Installation. Phenomenology.

\section{INTRODUCTION}

'The Time Machine' is a multi-screen, highperformance, generative video art installation based around multiple low cost computer platforms.

\begin{abstract}
"Clocks and calendar are necromantic devices tools by which the dead think for the living, and the dead's thoughts deflect the living's attention from the cycles in the present. This is a consequence of the mediation of cognition by artifacts, and it is a feature of how artifacts can distribute cognitive models across time, culture and space" (Birth 2012).
\end{abstract}

\section{GENERATIVE VIDEO}

The work uses looping time-lapse video shot in locations around the world to engage the viewer with a discussion on the experience, rhythm, repetition and flow of time. Running across multiple monitor screens the installation senses the audience and in response creates palindromic video loops from high resolution time-lapse video. The video feeds, recorded from around the world, tick and tock backward and forward creating a polyrhythmic, multi-screen time-piece, a videoclock locked in receptive, slowly evolving loops. A Time Machine reflecting the world. The backward and forth looping of the video feeds engage the viewer with both the re-assurance and the discomfort of seeing the world as 'clock-time'. The mechanistic vision that time is something created and measured, governed and ruled externally to ourselves and external to our experience.

The piece "The Time Machine" is a companion piece to the successful 2016 dual screen generative installation "What Do We Know Of Time When All We Can Know For Real Is Now?" (Buzzo 2016). Exhibited internationally at events such as 'Digital Futures' with British Computer Society and the Victoria and Albert Museum (London), Computer Art Congress 5, Paris and ACM Multimedia at OBA in Amsterdam the work comes from extensive investigation in the lived experience and perception and representations of time.

The work "The Time Machine" contrasts the external, machine perception of time with our internal, phenomenological experience of it. The notion of 'clock time' is a powerful and extremely widely adopted metaphor for what can be argued as the most fundamental element of experience (Munn 1992). Time links all things we see and perceive, from our earliest awareness of our own physical growth and mortality to more subtle realizations of the narrative procession of events 
and even the concept of causality (Garcia \& Prender 2014). The complexity of dissembling what this experience means has been wrestled with for millennia, as Augustine of Hippo asked in 400AD

"What then is time? If no one asks me, I know: if I wish to explain it to one that asketh, I know not."

The evidence and the balance of the philosophical argument is for procession and flow. What Heraclitus, and subsequently Nietsche described as all is chaos and becoming. However, clock time, an external mechanical, industrial notion of time, has become dominant since the turn of the last century. (Martineau 2015) The patterns and rhythms seen are considered cyclic, oscillating and reciprocating like the cogs and gears in a clock. Even the movements of stars, moon and planets around us are considered as an orrery, a child's instructional toy to describe the universe.

This work presents this mechanical clock fiction direct to the viewer. Folding half a dozen different types of time together in a multi-screen video form. Time lapse video from different time zones shifted and collated together, sunshine alongside moon light, dawn next to the falling of dusk. The video loops back and forth on each screen of the installation, creating a large polyrhythmic clock of high definition, full colour motion. Each screen detailing a passage of time from around the world, captured, frozen, forward and reverse. The timelapse loops slowly switch, selected from over a thousand separate pieces by generative algorithms on each host computer. Creating a slowly evolving and changing time machine. Gently rocking back and forth with a myriad of sub cadences, confronting the viewer with the unanswerable challenge of comprehending time.

\subsection{Technical requirements}

The Time Machine generative video installation utlilises 12 separate monitor and computer pairs. Each independently sensing the environment around them and dynamically controlling and presenting palindromic (looping back and forth) time lapse video shot on locations around the world. Screens and computers can be supplied. The installation therefore requires 24240 Volts AC power sources. Due to the low power requirements of the screens and computers there are no special requirements for power delivery other than access to sufficient mains electricity points. Multi-gang extension blocks are an ideal solution for power requirements.

\section{Setup one: In the round}

The installation of computers and screens can be adapted to suit available space but the arrangement is commonly 'in the round', i.e. the screens and monitors are bunched together allowing visitors and viewers of the work to view from 180-360 degrees of the installation. With the screens addressing all sides of an installation space. This commonly uses a floor space of approximately $2 \mathrm{~m} \times 2 \mathrm{~m}$.

\section{Setup two: Flat}

If space or arrangement is a suitable the work can also be exhibited 'flat' on a single wall with each screen arranged in an interlocking grid. If this option if requested, then suitable wall fixings or stands will need to be arranged to support all 12 screens.

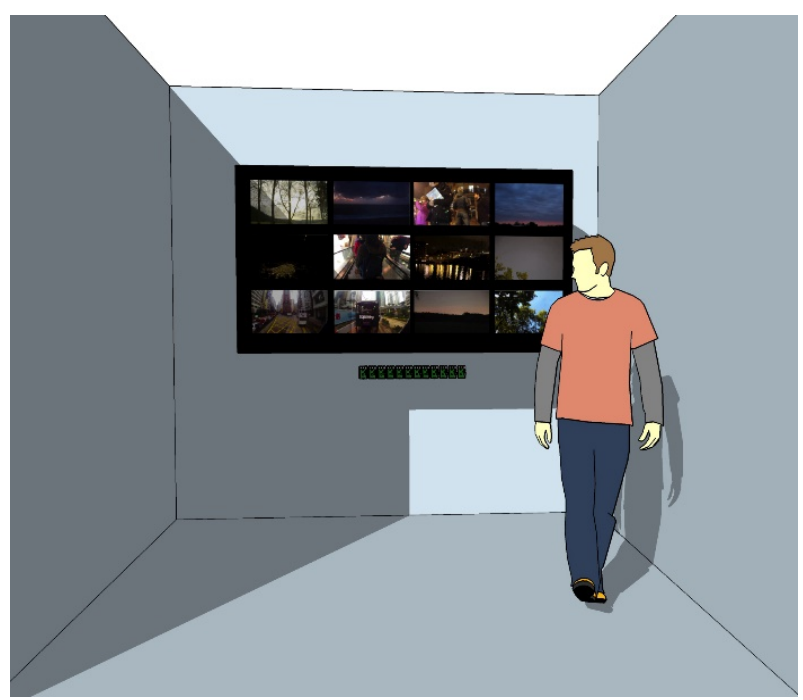

Figure 1: Standard 'flat' installation using matrix of 12 monitors showing Raspberry Pi driving computers.

\subsection{Technical background to the work}

When approaching the technical architecture for 'The Time Machine', the latest in a series of generative video art installations, for purposes of both cost and flexibility, the computing platform required to run a multi-screen installation required great thought. Previous generative or interactive multiscreen works had required highperformance and subsequently high cost computer platforms to run software and reproduce high frame-rate, high-quality video. For the latest piece of media art work, The Time Machine, based around extensive, high quality, time-lapse video material, the intention to run the installation on as many as 16 separate screens, and therefore computers, simultaneously, forced a rethink of the choice of platforms. Previous works had utilised high performance Macintosh mini-computers running custom software written in $\mathrm{C}++$. These platforms performed well with both video CODEC and software execution, enabling efficient high frame-rate playback of premium quality video material and near seamless generative or interactive functionality. 
When considering a new installation work the cost of including significant numbers of these highperformance but high-cost computing platforms was prohibitive, running into tens of thousands of pounds for computing hardware alone. To enable the production of the proposed new work alternative platforms and modes of delivery needed to be considered. After numerous comparisons and testing the Raspberry $\mathrm{Pi}$ (www.raspberrypi.org) low-cost, high-performance computer was selected as the platform of choice. Having a price advantage of approximately $20 \times$ versus previous high-cost computer platforms and the ability to execute custom C++ applications this hugely successful low-cost platform gave significant advantages over other devices. With a small form factor, low power consumption and a wide range of supporting software and peripherals the Raspberry Pi platform provides great opportunities for low cost, high performance custom media art installations.

Background to this work details the challenges, decisions and subsequent production processes behind the making of a new high performance, generative, multiscreen video installation using many interlinked Raspberry $\mathrm{Pi}$ mini-computers. Giving insights into artistic, design and technical decisions the paper contributes to both the creative process of artistic computer visualisation but also of the technical negotiations that must be undertaken when repurposing new technologies toward artistic goals.

The C++ application library openFrameworks (http://openframeworks.cc) was used as the starting point for programming using the Xcode Integrated Development environment (IDE) on MacOS computers to generate core code. This code was then ported by SSH remote connection to wifi connected Raspberry $\mathrm{Pi}$ target platforms for testing and native compilation. The extensive amount of video assets was managed in Aperture media library on MacOS and edited and colour graded using Final Cut Pro. The selection and editing process was extremely labour intensive both in the selection process for clips and the editing process.

The time-lapse video clips contained in the project were shot over a period of approximately 36 months across many locations including UK,
France, Portugal, Netherlands, Belgium, Hong Kong, Korea, Toronto and California. Primary video capture equipment included Canon 5D Mkll and EOS-M DSLR cameras running Magic Lantern firmware modifications and HerolV GoPro video systems. Assembly, selection and processing was primarily via MacOS with text code executed on MacMini computers.

\section{REFERENCES}

Birth, K. K. (2012) Objects of time: How things shape temporality. Culture, mind, and society. Palgrave Macmillan.

Buzzo, D. (2016) What do we Know of Time when all we can Know for Real is Now. In Proceedings of the $5^{\text {th }}$ Computer Art Congress, Paris, France, Europia Press. http://buzzo.com/what-do-we-knowof-time-when-all-we-can-know-for-real-is-now/ (accessed 24 May 2017).

Buzzo, D. and Jonas, D. (2015) Designing for the Impossible: Creating a Mobile Application for Time Dilation. In K. Ng, J. P. Bowen, \& N. Lambert (eds.), Electronic Visualisation and the Arts (EVA London 2015), pp. 178-185. London: BCS, Electronic Workshops in Computing. DOI: 10.14236/ewic/EVA2016.35

Garcia, T. and Pender, K. (2014) Another Order Of Time: Towards a Variable Intensity of the Now. Parrhesia: A Journal of Critical Philosophy, (19), pp. 1-13.

Husserl, E. (1991) On the Phenomenology of the Consciousness of Internal Time (1893-1917). In Edmund Husserl Collected Works.

Martineau, J. (2015) Time, Capitalism and Alienation. Brill.

McTaggart, J. E. (1908) The Unreality of Time. Mind: A Quarterly Review of Psychology and Philosophy, 17, pp. 456-473.

Munn, N. (1992) The Cultural Anthropology of Time: A Critical Essay. Annual Review of Anthropology, 21(1), pp. 93-123. 